\title{
The role of endoplasmic reticulum stress and the unfolded protein response in fibrosis
}

\author{
Stefania Lenna and Maria Trojanowska \\ Arthritis Center, Boston University School of Medicine, Boston, Massachusetts, USA
}

\begin{abstract}
Purpose of review-To review the present knowledge of the role of endoplasmic reticulum (ER) stress and unfolded protein response (UPR) in the pathogenesis of fibrotic diseases.

Recent findings-ER stress and UPR occur in a number of diseases associated with organ fibrosis; however, the contribution of these pathways to the fibrotic process has not been systematically investigated. Current studies suggest that prolonged ER stress may lead to fibrosis through activation of CCAAT/enhancer-binding homologous protein-mediated apoptosis, followed by an inflammatory response and release of profibrotic cytokines. A direct profibrotic role of UPR mediators in activation of TGF- $\beta$ signaling has been shown in lung fibroblasts. In addition, activation of ER stress and UPR pathways in immune cells contributes to increased production of proinflammatory cytokines.
\end{abstract}

Summary-Although limited in scope, current studies strongly suggest that ER stress and UPR may play an important role during development of fibrosis. Further studies are warranted to gain additional insights into the relationship between these processes.

\section{Keywords}

apoptosis; CHOP; ER stress; fibrosis; TGF- $\beta$; UPR

\section{INTRODUCTION}

Endoplasmic reticulum (ER) stress and unfolded protein response (UPR) constitute a homeostatic response to accumulation of misfolded protein as well as numerous other stimuli and can occur in various tissues and organs. Although ER stress serves a protective role that allows cells to deal with the noxious stimuli, prolonged ER stress contributes to the development and progression of many diseases, including neurodegenerative disorders, type II diabetes, artherosclerosis, and cancer (reviewed in [1]). Furthermore, selective signaling pathways that mediate ER stress can also contribute to other ER stress-independent cellular responses such as the well described regulation of innate immune signaling by the InositolRequiring Enzyme 1 (IRE1)/X-box binding protein 1 (XPB1) branch of UPR [2]. Whereas fibrosis is an integral part of many of the pathological conditions associated with ER stress and UPR, the role of these pathways in the fibrotic process is only beginning to emerge. This article will discuss current evidence supporting the role of ER stress in the process of fibrosis.

\section{(C) 2012 Wolters Kluwer Health | Lippincott Williams \& Wilkins}

Correspondence to Maria Trojanowska, Boston University School of Medicine, 72 East Concord St, E-5, Boston, MA 02118, USA. Tel: +1 617638 4318; fax: +1 617638 5226; trojanme@bu.edu.

Conflicts of interest

There are no conflicts of interest. 


\section{ENDOPLASMIC RETICULUM STRESS AND UNFOLDED PROTEIN RESPONSE: OVERVIEW}

The ER is an intracellular organelle in which most of the secretory and membrane proteins are synthesized, post-translationally modified, and folded into their correct conformations. Only properly folded proteins can be transported to the Golgi for further processing [3]. In addition, the ER is responsible for intracellular calcium homeostasis [4]. ER stress occurs when there is an imbalance between protein load and folding capacity, but can also be induced by other mechanisms [5]. In response to ER stress, cells activate an adaptive mechanism known as UPR [6]. UPR restores protein homeostasis by suppressing protein translation, inducing ER-related molecular chaperones to promote refolding of unfolded proteins, removing unfolded proteins by activating the ER-associated protein degradation (ERAD) system, and promoting cell survival. However, during prolonged or overwhelming ER stress when UPR fails to restore the normal function of the ER, a proapoptotic pathway is initiated through the activation of CCAAT/enhancer-binding homologous protein (CHOP) also termed growth-arrest and DNA-damage inducible gene 153 (GADD153) [7].

There are three branches of UPR that are initiated by distinct ER stress transducers located on the ER membrane: PKR-like endoplasmic reticulum kinase-(PERK), protein kinase $R$ (PKR)-like endoplasmic reticulum kinase; IRE1, inositol-requiring enzyme 1; and ATF6, activating transcription factor 6 . Under basal conditions these proteins are bound by the ER chaperone BiP (immunoglobulin-heavy-chain binding protein, also known as GRP78) and maintained in an inactive state [8]. When ER stress develops, BiP is sequestered by the misfolded peptides and, as a consequence, released from the three sensor proteins, which triggers activation of the UPR branches [9] All three branches of the UPR regulate the activation of CHOP, a central mediator of ER stress-induced apoptosis; however, activating transcription factor 4 (ATF4) is considered to be a major inducer of CHOP expression. CHOP is expressed at a very low level under physiological conditions, but its expression level is significantly increased in the presence of severe or persistent ER stress. As a transcription factor, $\mathrm{CHOP}$ has been shown to regulate numerous proapoptotic and antiapoptotic genes, including Bcl-2 and GADD34 [10] (Fig. 1).

\section{The PERK/elF2a/ATF4 pathway}

When unfolded proteins accumulate in the ER lumen, the first response is to attenuate further protein translation, which reduces the ER load and prevents accumulation of unfolded protein. The PERK/eukaryotic initiation factor $2 \alpha$ (eIF2 $\alpha$ ) pathway mediates this response. PERK, a transmembrane serine/threonine kinase localized in the ER membrane, is activated by ER stress via dimerization and autophosphorylation, leading to phosphorylation of eIF2 $a$ and resulting in a global inhibition of translation. Phosphorylated eIF2a promotes expression of the selected proteins such as transcription factors ATF4. In response to longterm adaptation to stress conditions, phosphorylation of eIF2a induces the expression of the growth arrest and DNA damage gene, GADD34, an important component of translational recovery during the ER stress response [11,12].

\section{The IRE1/XBP1 pathway}

During ER stress, IRE1 dissociates from BiP, becomes activated, and induces splicing of $\mathrm{XBP} 1$. The newly generated spliced XBP1 is an active transcription factor, which can induce downstream genes, such as ER chaperones and proteins involved in ERAD. These proteins work together to restore the ER homeostasis and promote cell survival [13,14]. 


\section{The ATF6 pathway}

ATF6 is a type II ER transmembrane protein. Like IRE1 and PERK, ATF6 binds to BiP and remains in an inactive state in unstressed cells. In response to ER stress, the BiP/ATF6 complex is dissociated, resulting in the translocation of ATF6 from the ER membrane to the Golgi in which it is cleaved by two serine proteases to produce the active form. The active ATF6 then moves to the nucleus and directly induces transcriptional activation of chaperone molecules such as BIP/GRP78, and ER stress response element (ERSE)-related genes through binding their promoters. It also induces other URP genes, such as XBP1 and CHOP $[15,16]$.

\section{KIDNEY FIBROSIS}

Activation of all three branches of the UPR has been demonstrated in a well established model of renal fibrosis, the unilateral urethral obstruction (UUO) model [17 $]$. The authors show that in this model prolonged ER stress leads to sustained activation of the ATF4 and the ATF6-CHOP proapoptotic pathways, as well as induction of other proapoptotic mediators, including c-Jun N-terminal kinases (JNK), Bax, and caspase 12. Although the IRE1 pathway also contributes to activation of the caspase 12 and JNK pathways [17 ], the unspliced and spliced levels of XBP1 decline, suggesting attenuation of the adaptive UPR response during this process. Activation of the apoptotic ER stress/UPR pathways results in apoptosis of tubular cells and subsequent renal fibrosis. Furthermore, this study shows that administration of the angiotensin II (Ang II) receptor blocker, candesartan, or an ER chemical chaperone, 4-phenylbutyric acid (4-PBA), resulted in amelioration of fibrosis, which correlated with suppression of the ATF4 and ATF6 pathways and restoration of XBP1 expression, suggesting that ER stress/UPR may play a causative role in this model. Given the prominent role of elevated Ang II in cardiac and renal disorders, the association of the Ang II pathway with ER stress/UPR is very intriguing; however, it is not clear at this time how these two pathways are interconnected.

In a model of diabetic nephropathy that used aged C57/Bl mice, the association of the ER stress-CHOP pathway with the development of the nephropathy was also described [18]. Furthermore, CHOP-deficient mice were protected from developing diabetic nephropathy. The authors present additional data in support of inflammation, and specifically TNF-a, in induction of the ER stress proapoptotic CHOP pathway in tubular interstitial cells.

\section{CARDIAC FIBROSIS}

In the heart, ER stress and UPR have been linked to ischemia-reperfusion injury, whereas a prolonged ER stress has been shown to contribute to cardiac hypertrophy and heart failure [19]. An early study showed that increased pressure load rapidly induces ER stress, whereas chronic perturbation of ER function, which in that study was induced by the overexpression of mutated Lysine-Aspartic acid-Glutamic acid-Leucine protein, led to cardiac dilation with congestive heart failure and interstitial fibrosis [20]. Several other studies using different experimental models have confirmed association of ER stress with heart hypertrophy and fibrosis [21]; however, it is important to note that depending on the context ER stress may play a pathological or a protective role in heart damage and failure [19]. Recent preclinical studies have shown that two US Food and Drug Administration (FDA)-approved chemical chaperones, 4-phenylbutyric acid (4-PBA) and tauroursodeoxycholic acid (TUDCA), showed very promising results in reducing ER stress in several animal models. The oral administration of 4-PBA markedly reduced hypertrophy and down-regulated fibrosis-related genes in a model of pressure-overload cardiac hypertrophy [22]. Similarly, 4-PBA administered subcutaneously prevented cardiac fibrosis induced by injection of isoproterenol [23]. Kassan et al. [24] have examined the effects of TUDCA and 4-PBA in the Ang II 
infusion model. Their study shows that Ang II infusion is associated with ER stress induction in the heart and that inhibition of ER stress reduced cardiac hypertrophy and fibrosis, which correlated with reduced TGF- $\beta$ activity and reduced oxidative stress in this model. Furthermore, the authors show that inhibition of ER stress improves vascular function in the aorta and in mesenteric arteries (MRAs), in part, by reducing TGF- $\beta$ activity in the aorta and by reducing oxidative stress in both aorta and MRAs.

\section{LIVER FIBROSIS}

The liver is one of the major secretory organs in the body with a key role in glucose and lipid metabolism, and a number of pathological stimuli, including ethanol, drugs, viral infection, and ischemia-reperfusion, have been shown to cause perturbation of the ER function and activation of the UPR pathways in hepatocytes (reviewed in [5]). Furthermore, ER stress/UPR has been implicated in the pathogenesis of several liver diseases, including nonalcoholic fatty liver disease, cholestasis, chronic viral hepatitis, alcohol-induced liver injury, and others [5]. Hepatic fibrosis is a final common pathway for almost all liver diseases resulting from activation of hepatic stellate cells (HSCs), a principal liver cell-type responsible for extracellular matrix production and scar tissue formation [25]. A link between UPR and liver fibrosis has been examined in a mouse model of a1-antitrypsin (a1AT) deficiency [26]. a1-AT deficiency is a genetic disorder caused by a mutation in the a1AT serine protease inhibitor gene, and primarily affects the liver and lungs. A common a1ATZ variant represents a single amino acid substitution that causes protein misfolding and accumulation in the ER. Transgenic mice expressing the human a1-ATZ variant (PiZ mice) develop mild symptoms of ER stress [27], but CHOP is not upregulated in these mice.

However, expression of the a1-ATZ variant predisposes these mice to develop full ER stress and UPR after additional insult such as cholestasis by bile duct ligation (BDL). Furthermore, it was shown that PiZ mice were more susceptible to liver fibrosis induced by cholestasis.

Augmented fibrosis correlated with upregulation of CHOP, increased hepatocyte apoptosis, and increased expression of TGF- $\beta$, as compared to wild-type mice. A key role of CHOP in promoting the fibrotic response was demonstrated in a related study that showed amelioration of cholestasis-induced liver fibrosis in CHOP-deficient mice [28]. As illustrated by these studies the CHOP-mediated apoptosis of hepatocytes is a primary mechanism linking ER stress and fibrosis in the liver, whereas HSCs do not develop ER stress but become activated in response to TGF- $\beta$ and other mediators, resulting from hepatocyte death. An interesting recent study has shown that ER stress can be induced in HSCs by cannabidiol (CBD). Furthermore, prolonged CBD-mediated ER stress caused apoptosis of activated but not quiescent HSCs [29]. The authors of the latter study postulate that CBD may be useful as a therapeutic agent in liver fibrosis.

Although a1-AT deficiency in the liver did not produce ER stress in a mouse model [26], in monocytes isolated from patients with the a1-AT deficiency (ZZ) activation of the ER stress/UPR pathways and increased production of proinflammatory cytokines both at baseline and after stimulation with lipopolysaccharides were observed [30]. This suggests that the role of the a1-ATZ variant in ER stress induction may depend on the cell type and may also be species-dependent.

\section{LUNG FIBROSIS}

The activation of ER stress and UPR was first observed in patients with familial interstitial pneumonia (FIP) carrying a mutation in surfactant protein C (SFTPC) [31]. The presence of UPR was demonstrated in type II alveolar epithelial cells, which specifically express SFTPC. In subsequent studies prominent ER stress in alveolar epithelial cells (AECs) was also observed in patients with FIP not carrying the SFTPC mutation, as well as patients with 
sporadic idiopathic pulmonary fibrosis (IPF) [32]. Interestingly, in a large proportion of those patients herpes virus protein expression was found to colocalize with UPR markers, suggesting that herpes viruses may induce ER stress in those patients [32]. Other potential causes of ER stress in the lungs may include exposure to inhaled particulate matter or cigarette smoke [33]. The link between ER stress, fibrosis, and viral infection was further investigated in mice infected with murine gamma herpes virus 68 (MHV68) [33]. It was shown that infection with MHV68 leads to development of ER stress in AECs, as well as AEC apoptosis and subsequent lung fibrosis in aged mice, whereas infected young mice develop mild reversible pneumonitis only. This study suggests that aging may lead to impairment of the UPR response, which, in turn, may contribute to the development of IPF in the elderly. To further investigate the role of mutated surfactant protein Lawson et al. [34] have developed a transgenic mouse expressing human mutant surfactant protein (L188Q SPFTC) exclusively in type II AECs by using the Tet-On system. These mice showed evidence of ER stress and UPR in AECs, but ER stress/UPR alone was not sufficient to induce lung fibrosis. However, exaggerated fibrosis was observed in the mutant mice after administration of bleomycin, suggesting that activation of ER stress plays a role in the severity of the fibrotic process [34]. The follow-up in-vitro studies have shown that expression of the L188Q mutant in AECs leads to induction of epithelial mesenchymal transition (EMT), suggesting that this process may also contribute to the ER stressdependent fibrosis in vivo [35].

A recent study by Baek et al. [36 $\left[6^{\square}\right]$ has also suggested that selected ER stress pathways may contribute to the TGF- $\beta$-induced myofibroblast differentiation and collagen production. The authors showed that TGF- $\beta$ induces BiP/GRP78, XPB-1 and ATF6a, but not $p$-eIF2 $\alpha$ or CHOP in human and murine pulmonary fibroblasts. Blockade of GRP78 by siRNA significantly reduced collagen and alpha smooth muscle actin expression in response to TGF- $\beta$, suggesting, for the first time, a profibrotic role of ER stress directly in fibroblasts. Furthermore, this study suggests that UPR pathways may be activated downstream of reactive oxygen species (ROS) induction and may contribute to the chronic ROS generation in fibroblasts [ $\left.36^{\square}\right]$. Since ROS have been implicated in the pathogenesis of pulmonary fibrosis, this study underscores the link between ER stress and ROS in the development of fibrosis.

\section{PULMONARY ARTERIAL HYPERTENSION}

Endoplasmic reticulum stress and UPR are prominently activated in endothelial cells and macrophages during the progression of atherosclerosis [37]. As discussed in the earlier section, ER stress is also linked to endothelial cell dysfunction in aorta and mesenteric arteries in the Ang-II infusion model [24]; however, relatively little is known about whether ER stress also contributes to the vascular dysfunction in pulmonary arterial hypertension (PAH). Endothelin-1 (ET-1) is a potent vasoconstrictor that plays pathological role in pulmonary hypertension. Yeager et al. [38] have investigated the link between ET-1 and ER stress in pulmonary arterial smooth muscle cells (PASMCs). They found that in those cells ET-1 induces UPR via the endothelin A receptor and ERK 1/2. This response was associated with induction of several proinflammatory cytokines in an ATFa-dependent manner. Interestingly, previous studies by Lenna et al. [39] have shown that ET-1 and IL-6 are induced in response to ER stress in endothelial cells, suggesting that ER stress and UPR could contribute to the upregulation of ET-1 and proinflammatory cytokines during pathogenesis of PAH. Importantly, it was shown that Human Leukocyte Antigen B35, which is associated with increased risk for developing PAH in Italian SSc patients [40], induced ER stress-mediated up-regulation of ET-1 [39]. Additional studies have shown that the ATF4 branch of UPR mediated up-regulation of ET-1 in endothelial cells (Keystone Symposium Fibrosis, http://www.keystonesymposia.org/meetings/viewMeetings.cfm? 
MeetingID=1154). Although these studies suggest that ER stress and UPR could contribute to PAH, it would be important to extend these findings to animal models of PAH, as well as analyses of lung specimens from patients with pulmonary hypertension. Autophagy, which is frequently associated with prolonged ER stress, is a cellular mechanism facilitating lysosome-dependent degradation of organelles and proteins [41]. It may be relevant that pulmonary hypertension is characterized by elevation of the autophagic protein LC3B both in humans and in a mouse model of hypoxia-induced pulmonary hypertension [42]. The main finding of the latter study suggests that autophagy plays a protective role in pulmonary hypertension, as mice deficient for LC3B or an activator of the LC3B gene, Egr-1, have worsened disease [42].

Inflammation plays an important, albeit not fully elucidated, role in PAH [43]. We have observed significantly elevated expression levels of ER stress and UPR markers in PBMCs isolated from patients with SSc-PAH in comparison to patients with limited SSc and healthy controls (Lenna et al., manuscript under revision). These initial studies suggest that ER stress and UPR may contribute to the pathogenesis of PAH by affecting endothelial and vascular smooth muscle cells, as well as immune cells, but further studies are warranted to confirm this possibility.

\section{CONCLUSION}

The contribution of ER stress and UPR to the pathogenesis of many diseases has been firmly established. With respect to fibrotic diseases, ER stress and UPR may play a role in promoting inflammation and release of TGF- $\beta$ in response to the CHOP-mediated activation of apoptotic pathways. There is also evidence that ER stress and UPR may have a direct role in the TGF- $\beta$ response and myofibroblast activation through induction of ROS; however, additional studies are needed to further extend these novel findings. As UPR may have both harmful and beneficial effects depending on the specific organ and conditions, a better understanding of the role of these pathways in specific fibrotic disorders is needed as a prerequisite for future therapeutic interventions, including chaperones to improve protein folding.

\section{Acknowledgments}

The authors' work has been supported by the NIH NIAMS grants RO1 AR42334, R01 AR44883, P50 AR060780, the Scleroderma Foundation and from GILS (Gruppo Italiano per la Lotta alla Sclerodermia).

\section{REFERENCES AND RECOMMENDED READING}

Papers of particular interest, published within the annual period of review, have been highlighted as:

$\square$ of special interest

प[ of outstanding interest

Additional references related to this topic can also be found in the Current World Literature section in this issue (p. 699).

1. Ozcan L, Tabas I. Role of endoplasmic reticulum stress in metabolic disease and other disorders. Annu Rev Med. 2012; 63:317-328. [PubMed: 22248326]

2. Martinon F, Glimcher LH. Regulation of innate immunity by signaling pathways emerging from the endoplasmic reticulum. Curr Opin Immunol. 2011; 23:35-40. [PubMed: 21094031]

3. Ron D, Walter P. Signal integration in the endoplasmic reticulum unfolded protein response. Nat Rev Mol Cell Biol. 2007; 8:519-529. [PubMed: 17565364] 
4. Krebs J, Groenendyk J, Michalak M. Ca2+-signaling, alternative splicing and endoplasmic reticulum stress responses. Neurochem Res. 2011; 36:1198-1211. [PubMed: 21365449]

5. Malhi H, Kaufman RJ. Endoplasmic reticulum stress in liver disease. J Hepatol. 2011; 54:795-809. [PubMed: 21145844]

6. Hetz C, Martinon F, Rodriguez D, Glimcher LH. The unfolded protein response: integrating stress signals through the stress sensor IRE1alpha. Physiol Rev. 2011; 91:1219-1243. [PubMed: 22013210]

7. Rao RV, Ellerby HM, Bredesen DE. Coupling endoplasmic reticulum stress to the cell death program. Cell Death Differ. 2004; 11:372-380. [PubMed: 14765132]

8. Bertolotti A, Zhang Y, Hendershot LM, et al. Dynamic interaction of BiP and ER stress transducers in the unfolded-protein response. Nat Cell Biol. 2000; 2:326-332. [PubMed: 10854322]

9. Hetz C. The unfolded protein response: controlling cell fate decisions under ER stress and beyond. Nat Rev Mol Cell Biol. 2012; 13:89-102. [PubMed: 22251901]

10. Kim R, Emi M, Tanabe K, Murakami S. Role of the unfolded protein response in cell death. Apoptosis. 2006; 11:5-13. [PubMed: 16374548]

11. Harding HP, Zeng H, Zhang Y, et al. Diabetes mellitus and exocrine pancreatic dysfunction in perk -/- mice reveals a role for translational control in secretory cell survival. Mol Cell. 2001; 7:11531163. [PubMed: 11430819]

12. Harding HP, Zhang Y, Bertolotti A, et al. Perk is essential for translational regulation and cell survival during the unfolded protein response. Mol Cell. 2000; 5:897-904. [PubMed: 10882126]

13. Calfon M, Zeng H, Urano F, et al. IRE1 couples endoplasmic reticulum load to secretory capacity by processing the XBP-1 mRNA. Nature. 2002; 415:92-96. [PubMed: 11780124]

14. Lee AH, Iwakoshi NN, Glimcher LH. XBP-1 regulates a subset of endoplasmic reticulum resident chaperone genes in the unfolded protein response. Mol Cell Biol. 2003; 23:7448-7459. [PubMed: 14559994]

15. Chen X, Shen J, Prywes R. The luminal domain of ATF6 senses endoplasmic reticulum (ER) stress and causes translocation of ATF6 from the ER to the Golgi. J Biol Chem. 2002; 277:1304513052. [PubMed: 11821395]

16. Yoshida H, Matsui T, Yamamoto A, et al. XBP1 mRNA is induced by ATF6 and spliced by IRE1 in response to ER stress to produce a highly active transcription factor. Cell. 2001; 107:881-891. [PubMed: 11779464]

170. Chiang CK, Hsu SP, Wu CT, et al. Endoplasmic reticulum stress implicated in the development of renal fibrosis. Mol Med. 2011; 17:1295-1305. This study thoroughly describes activation of ER stress and UPR during renal fibrosis. [PubMed: 21863214]

18. Wu J, Zhang R, Torreggiani M, et al. Induction of diabetes in aged C57B6 mice results in severe nephropathy: an association with oxidative stress, endoplasmic reticulum stress, and inflammation. Am J Pathol. 2010; 176:2163-2176. [PubMed: 20363923]

19. Glembotski CC. The role of the unfolded protein response in the heart. J Mol Cell Cardiol. 2008; 44:453-459. [PubMed: 18054039]

20. Hamada H, Suzuki M, Yuasa S, et al. Dilated cardiomyopathy caused by aberrant endoplasmic reticulum quality control in mutant KDEL receptor transgenic mice. Mol Cell Biol. 2004; 24:8007-8017. [PubMed: 15340063]

21. Dickhout JG, Carlisle RE, Austin RC. Interrelationship between cardiac hypertrophy, heart failure, and chronic kidney disease: endoplasmic reticulum stress as a mediator of pathogenesis. Circ Res. 2011; 108:629-642. [PubMed: 21372294]

22. Park CS, Cha H, Kwon EJ, et al. The chemical chaperone 4-phenylbutyric acid attenuates pressureoverload cardiac hypertrophy by alleviating endoplasmic reticulum stress. Biochem Biophys Res Commun. 2012; 421:578-584. [PubMed: 22525677]

23. Ayala P, Montenegro J, Vivar R, et al. Attenuation of endoplasmic reticulum stress using the chemical chaperone 4-phenylbutyric acid prevents cardiac fibrosis induced by isoproterenol. Exp Mol Pathol. 2012; 92:97-104. [PubMed: 22101259]

240. Kassan M, Galan M, Partyka M, et al. Endoplasmic reticulum stress is involved in cardiac damage and vascular endothelial dysfunction in hypertensive mice. Arterioscler Thromb Vasc Biol. 2012; 32:1652-1661. This work demonstrates the efficacy of 'chemical chaperones', PBA, and 
TUDCA in ameliorating harmful effects of prolonged ER stress, suggesting a possibility of an anti-ER stress therapy. [PubMed: 22539597]

25. Brenner DA. Molecular pathogenesis of liver fibrosis. Trans Am Clin Climatol Assoc. 2009; 120:361-368. [PubMed: 19768189]

26. Mencin A, Seki E, Osawa Y, et al. Alpha-1 antitrypsin Z protein (PiZ) increases hepatic fibrosis in a murine model of cholestasis. Hepatology. 2007; 46:1443-1452. [PubMed: 17668872]

27. Carlson JA, Rogers BB, Sifers RN, et al. Accumulation of PiZ alpha 1-antitrypsin causes liver damage in transgenic mice. J Clin Invest. 1989; 83:1183-1190. [PubMed: 2784798]

28. Tamaki N, Hatano E, Taura K, et al. CHOP deficiency attenuates cholestasis-induced liver fibrosis by reduction of hepatocyte injury. Am J Physiol Gastro-intest Liver Physiol. 2008; 294:G498-505.

29. Lim MP, Devi LA, Rozenfeld R. Cannabidiol causes activated hepatic stellate cell death through a mechanism of endoplasmic reticulum stress-induced apoptosis. Cell Death Dis. 2011; 2:e170. [PubMed: 21654828]

30. Carroll TP, Greene CM, O'Connor CA, et al. Evidence for unfolded protein response activation in monocytes from individuals with alpha-1 antitrypsin deficiency. J Immunol. 2010; 184:45384546. [PubMed: 20228200]

31. Thomas AQ, Lane K, Phillips J 3rd, et al. Heterozygosity for a surfactant protein C gene mutation associated with usual interstitial pneumonitis and cellular nonspecific interstitial pneumonitis in one kindred. Am J Respir Crit Care Med. 2002; 165:1322-1328. [PubMed: 11991887]

32. Lawson WE, Crossno PF, Polosukhin VV, et al. Endoplasmic reticulum stress in alveolar epithelial cells is prominent in IPF: association with altered surfactant protein processing and herpesvirus infection. Am J Physiol Lung Cell Mol Physiol. 2008; 294:L1119-1126. [PubMed: 18390830]

33. Tanjore H, Blackwell TS, Lawson WE. Emerging evidence for endoplasmic reticulum stress in the pathogenesis of idiopathic pulmonary fibrosis. Am J Physiol Lung Cell Mol Physiol. 2012; 302:L721-L729. [PubMed: 22287606]

34. Lawson WE, Cheng DS, Degryse AL, et al. Endoplasmic reticulum stress enhances fibrotic remodeling in the lungs. Proc Natl Acad Sci U S A. 2011; 108:10562-10567. [PubMed: 21670280]

35. Tanjore H, Cheng DS, Degryse AL, et al. Alveolar epithelial cells undergo epithelial-tomesenchymal transition in response to endoplasmic reticulum stress. J Biol Chem. 2011; 286:30972-30980. [PubMed: 21757695]

360. Baek HA, Kim DS, Park HS, et al. Involvement of endoplasmic reticulum stress in myofibroblastic differentiation of lung fibroblasts. Am J Respir Cell Mol Biol. 2012; 46:731739. This work is a first demonstration of the activation of selected UPR pathways by TGFb in myofibroblasts. [PubMed: 21852685]

37. Tabas I. The role of endoplasmic reticulum stress in the progression of atherosclerosis. Circ Res. 2010; 107:839-850. [PubMed: 20884885]

38. Yeager ME, Belchenko DD, Nguyen CM, et al. Endothelin-1, the unfolded protein response, and persistent inflammation: role of pulmonary artery smooth muscle cells. Am J Respir Cell Mol Biol. 2012; 46:14-22. [PubMed: 21778413]

39. Lenna S, Townsend DM, Tan FK, et al. HLA-B35 upregulates endothelin-1 and downregulates endothelial nitric oxide synthase via endoplasmic reticulum stress response in endothelial cells. J Immunol. 184:4654-4661. [PubMed: 20335527]

40. Grigolo B, Mazzetti I, Meliconi R, et al. Antitopoisomerase II alpha autoantibodies in systemic sclerosis-association with pulmonary hypertension and HLA-B35. Clin Exp Immunol. 2000; 121:539-543. [PubMed: 10971522]

41. Mizushima N, Levine B, Cuervo AM, Klionsky DJ. Autophagy fights disease through cellular selfdigestion. Nature. 2008; 451:1069-1075. [PubMed: 18305538]

42. Lee SJ, Smith A, Guo L, et al. Autophagic protein LC3B confers resistance against hypoxiainduced pulmonary hypertension. Am J Respir Crit Care Med. 2011; 183:649-658. [PubMed: 20889906]

43. Schermuly RT, Ghofrani HA, Wilkins MR, Grimminger F. Mechanisms of disease: pulmonary arterial hypertension. Nat Rev Cardiol. 2011; 8:443-455. [PubMed: 21691314] 


\section{KEY POINTS}

- ER stress and UPR have been observed in lung, liver, and kidney fibrosis.

- CCAAT/enhancer-binding homologous protein (CHOP)/growth-arrest and DNA-damage inducible gene 153 (GADD153) is a key mediator of ER stressinduced proapoptotic pathways.

- CCAAT/CHOP-deficient mice are protected from the ER stress-induced cell death, as well as fibrosis.

- Individual branches of the UPR are also involved in other cellular processes such as inflammation or TGF- $\beta$ signaling. 


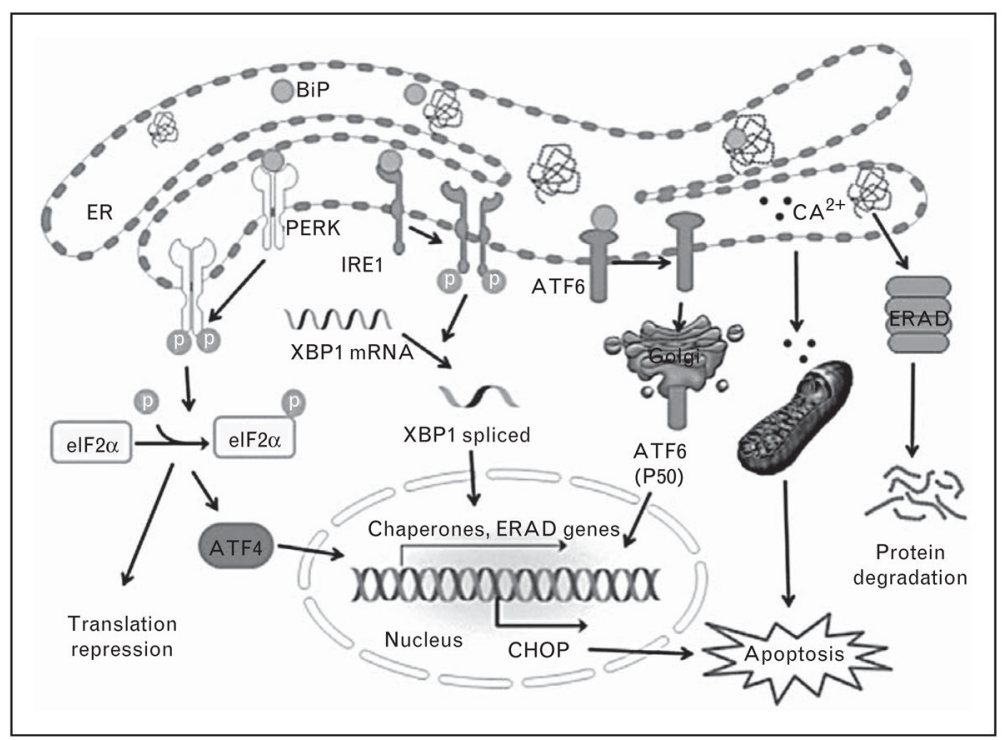

FIGURE 1.

Signaling pathways of the UPR. Accumulation of unfolded proteins in ER lumen results in the ER stress. In response to ER stress, $\mathrm{BiP}$ dissociates from the ER stress transducers and binds to unfolded and misfolded proteins, resulting in the activation of the ER stress transducers: PERK, IRE1, and ATF6. Activation of PERK increases phosphorylation of eIF2a, leading to attenuation of protein synthesis and an increase in ATF4 translation. During prolonged stress, ATF4 can induce CHOP, a proapoptotic transcription factor. Once activated, IRE1 splices XBP1 producing the active form XBP1-S (spliced XBP1), which upregulates ER chaperones and proteins implicated in the ER-associated protein degradation (ERAD). ATF6 translocates to Golgi, in which it is activated by proteolysis. Activated ATF6 transcriptionally induces ERAD genes and upregulates CHOP expression. ER, endoplasmic reticulum; UPR, unfolded protein response. 